\title{
Energy Efficiency in Energy Harvesting Cooperative Networks with Self-Energy Recycling
}

\author{
Shiyang $\mathrm{Hu}^{1}$, Zhiguo Ding ${ }^{1}$, Qiang $\mathrm{Ni}^{1}$, Wenjuan $\mathrm{Yu}^{1}$ and Zhengyu Song ${ }^{2}$ \\ ${ }^{1}$ School of Computing and Communications, Lancaster University, LA1 4WA, UK \\ ${ }^{2}$ School of Information and Electronics, Beijing Institute of Technology, 100081, Beijing, China
}

\begin{abstract}
Cooperative communication has been identified as an important component in the $5 \mathrm{G}$ system. This paper considers a decode-and-forward (DF) relaying wireless cooperative network, in which the self-energy recycling relay is powered by radiofrequency (RF) signal from the source and its transmitted power from the loop-back channel. The harvested energy is used to support the relay transmissions. Based on a self-energy recycling relaying protocol, we study the optimization of energy efficiency in wireless cooperative networks. Although the formulated optimization problem is not convex, it can be re-constructed to a parametric problem in the convex form by using the non-linear fractional programming, to which closed form solutions can be found by using the Lagrange multiplier method. The simulation results are presented to verify the effectiveness of this solution proposed in this paper.
\end{abstract}

\section{INTRODUCTION}

Wireless energy harvesting is a promising approach to charge batteries in the future $5 \mathrm{G}$ wireless communication network [1]-[3]. For many extreme conditions, the traditional power supplies are impossible to recharge or to replace [1]. For example, more and more medical devices are implanted under the patient's skin for recording body data. These devices are difficult and costly to replace the battery. Therefore, wirelessly recharging the battery from the external source has a high demand, which motivates the energy harvesting technology emerging. Some works pointed out that the power of solar, wind and thermoelectric phenomena can be used as the external sources to wirelessly charge [4]-[7]. Besides, the radio-frequency (RF) signals as a potential way to achieve the wireless energy harvesting has drawn the considerable attention [8].

However, the practical circuit limits the development of simultaneously receiving information and harvesting energy at the receiver node. Hence a more practical design was proposed in [3], where an energy harvesting node performs information decoding and energy harvesting separately according to the time switching protocol or the power splitting protocol. Some works studied the new energy harvesting protocols for pointto-point communication networks [1], [8]-[10]. Besides, the authors in [11] researched the time switching protocol and the power splitting protocol in wireless cooperative networks. Specifically, in [11], the authors employed energy harvesting protocol in an amplify-to-forward (AF) cooperative network and studied the outage probability and the throughput in the system. The authors in [12] studied power allocation strategies in a cooperative network with one energy harvesting relay and multiple pairs of sources and destinations.

The half-duplex cooperative network is extensively studied in the wireless energy harvesting study. There are some researchers focusing on the full-duplex structure study in the energy harvesting. The full-duplex wireless powered networks based on time-switching protocol were studied in [13], [14]. In the full-duplex wireless powered network, the node is capable of transmitting energy and receiving information simultaneously. In [15], a new self-energy recycling protocol was proposed. The self-energy recycling relaying protocol is based on a two-phase transmission protocol. The full-duplex structure relay is equipped with two antennas. It receives the information from the source node in the first phase. In the second phase, the energy harvesting relay uses its receiving antenna to collect power from the source and uses its transmission antenna to relay the decoded information to the destination node. The advantage of this protocol is that the energy harvesting relay not only harvests power form the source node, but also reuses the energy from its transmitted power by its loop-back channel. This protocol was set up in a MISO relaying channel in [15]

Motivated by this, we study energy-efficiency maximization in decode-and-forward (DF) wireless cooperative network with the self-energy recycling protocol. The concept of energyefficient study in wireless communication has drawn much attention recently [16]-[18]. Solutions of the energy efficiency (bit-per-Joule) optimization problem can ensure that wireless communication systems utilize energy in a more environmentfriendly way. The energy-efficient maximization problem is defined as a ratio of the channel capacity and overall power consumption in the block time. Particularly the energy-efficient maximization problems are formulated based on the selfenergy recycling protocol. The formulated optimization problem is not in standard convex form, but can be transformed to a parametric problem in the convex form by applying non-linear fractional programming. The re-formulated problems can be solved by applying the Lagrange multiplier method and the gradient method. Simulation results are provided to verify that the proposed algorithm improves energy utilization compared to the case without applying energy-efficient optimization. The trade-off between the energy efficiency and system parameters is also analysed by using the provided numerical results.

The rest of this paper is organized as follows. Section II presents the system model of an energy harvesting wireless cooperative network. Section III presents the details for the transmission model based on the self-energy recycling protocol and formulates the corresponding energy-efficient problem. Section IV presents the solutions to transform original problems into solvable convex optimization form. The numerical results are provided in $\mathrm{V}$ and section VI concludes this paper. 


\section{System MODEL}

The wireless cooperative network considered here includes one source-destination pair and one energy harvesting relay. The source node and the destination node are equipped one antenna respectively. The relay node has two antennas, one antenna is used for information transmission while the other one is used for receiving. We assume that there is no direct link between the source node and the destination node, i.e. the source node intends to transmit message to the destination node with the assist of the relay node. Channels are modelled as quasi-static block fading channels. The perfect channel state information is available at the destination node.

The energy harvesting relay is solely powered by the source. It can harvest energy from the source node and utilize the energy to relay the source information. This assumption is used in [11]. The battery capacity of the energy harvesting relay is assumed as infinite. The decode-and-forward (DF) scheme is employed in the cooperative network. A new energy harvesting protocol with self-energy recycling relay is considered in this paper. The detailed analysis based on this protocol is given in the following sections. We also assume that at the relay, the power consumed to process the harvested energy is negligible, when compared to the power used in transmitting information to the destination.

\section{ENERGY EFFICIENCY WITH SELF-ENERGY RECYCLING RELAY}

In this section, we study the energy-efficient optimization problem in self-energy recycling relay networks. The transmission model is given as follows.

\section{A. Relay Protocol and Transmission Model}

The whole transmission process is operated in the block time, denoted by $T$. Without loss of generality, $T$ is normalized to be unity. The information transmission process is split into two phases. In the first phase, the source transmits information to the relay for $T / 2$ time and relay uses its receiving antenna to receive information. In the second phase, the source transmits RF signals to power the energy harvesting relay and the relay sends decoded information to the destination. Recall that the energy harvesting relay is equipped with one transmission antenna and one receiving antenna, therefore it is capable of relaying the information and collecting energy simultaneously.

The energy harvesting relay first receives the information from the source with its receiving antenna. The received signal at the relay can be expressed as

$$
y_{r}^{1}=\sqrt{P_{s}} h x_{s}+n_{r}
$$

where $P_{s}$ is the transmitted power, $h$ is the channel gain from the source to the receiving antenna of the relay, and $x_{s}$ is the normalized transmitted signal with unit power and $n_{r}$ is the baseband additive white Gaussian noise (AWGN) from the receiving antenna.

In the second phase, we assume that the energy harvesting relay can decode the source message successfully. The relay uses its transmission antenna to relay the information to the destination node. The received signal at the destination is given by

$$
y_{d}=\sqrt{P_{r}} g \bar{x}_{s}+n_{d}
$$

where $P_{r}$ is the power transmitted from the relay, $g$ is the channel gain between the transmission antenna of the relay and the destination node, $\bar{x}_{s}$ is the decoded signal for the destination and $n_{d}$ is the AWGN from the receiving antenna. Concurrently, the energy harvesting relay is wireless powered by the source node with dedicated energy-bearing signal. The received signal at the relay is

$$
y_{r}^{2}=\sqrt{P_{s}} h x_{e}+\sqrt{P_{r}} f \bar{x}_{s}+n_{r}+n_{c},
$$

where $x_{e}$ is normalized signal with unit power transmitted by the source node, $f$ is the channel gain of the loop channel at the relay node, and $n_{c}$ is the sampled AWGN from the RF band to baseband conversion [11]. The relay node not only collects energy from the source node, but also recycles part of its transmitted power due to its two antenna being activated at the same time. In the other full-duplex relaying structure, the relay employs interference cancellation techniques to eliminate the loop-back interference signal. In the energy harvesting cooperative network, the loop-back signal can be reused at the relay as the transmitted power. The harvest energy is given by

$$
E=\eta\left(P_{s}|h|^{2}+P_{r}|f|^{2}\right) \frac{T}{2}
$$

where $\eta$ is the energy conversion efficiency coefficient. Then the transmitted power at the relay node is $\frac{E}{T / 2}$ which can be expressed as

$$
P_{r}=\frac{\eta P_{s}|h|^{2}}{1-\eta|f|^{2}},
$$

In the case of the cooperative network using DF protocols without the direct link between the source and the destination, the channel capacity can be calculated as

$$
C=\min \left(C_{s r}, C_{r d}\right)
$$

where $C_{s r}$ and $C_{r d}$ are the channel capacity from the source to the relay and from the relay to the destination respectively. $C_{s r}$ and $C_{r d}$ can be express as

$$
C_{s r}=\frac{1}{2} \log \left(1+\frac{P_{s}|h|^{2}}{\sigma_{r}^{2}}\right),
$$

and

$$
C_{r d}=\frac{1}{2} \log \left(1+\frac{\eta P_{s}|h|^{2}|g|^{2}}{\left(1-\eta|f|^{2}\right) \sigma_{d}^{2}}\right),
$$

where $\sigma_{r}^{2}$ and $\sigma_{d}^{2}$ are the variances of the $n_{r}$ and $n_{d}$.

\section{B. Problem Formulation}

In this section, we consider the energy efficiency problem for the considered network. The energy efficiency is a ratio between the system channel capacity and the overall power consumption in one transmission block time. It is assumed that each node has a constant circuit power consumption for signal processing, which is independent of the power used for transmitting signal or the harvested power. We denote $P_{1}, P_{2}$ 
and $P_{3}$ by the circuit consumed power in the source, the relay and the destination, respectively.

According to the above relaying protocol, nodes are activated in the corresponding transmission phase. Therefore the total consumed power in this wireless cooperative network is given by

$$
P_{c}=P_{1}+P_{2}+\frac{1}{2} P_{3}+P_{s},
$$

This work aims to maximize the energy efficiency in the considered model. The optimization problem is given by

$$
\begin{array}{ll} 
& \max _{P_{s}} \frac{C}{P_{c}} \\
\text { s.t. } & C 1: P_{s} \leq \overline{P_{s}}, \\
& C 2: C \geq \bar{C}, \\
& C 3: P_{s} \geq 0 .
\end{array}
$$

where the predefined variable $\overline{P_{s}}$ is the maximum transmit power limitation at the source. $\bar{C}$ is the minimum required channel capacity in order to meet the QoS criterion.

One can easily verify that the proposed problem above is not a convex optimization problem, because the objective function is a ratio of the channel capacity and total consumed power. The solution to this formulated problem is given in the following section.

\section{ENERGy-EFFICIENT POWER Allocation}

The proposed problem is not in the standard convex form. However the problem can be transformed to a parametric optimization problem by exploring the properties of the nonlinear fractional programming [19]. According to the parametric method, we introduce a new variable $q^{*}$ to denote the optimum energy efficient for the proposed problem. $q^{*}$ can be expressed as

$$
\begin{aligned}
q^{*} & =\frac{C^{*}}{P_{c}{ }^{*}}, \\
& =\max _{P_{s}} \frac{C}{P_{c}} .
\end{aligned}
$$

Therefore, the transformed parametric problem is given by

$$
\begin{array}{ll} 
& \max _{P_{s}} C-q^{*} P_{c} \\
\text { s.t. } & C 1: P_{s} \leq \overline{P_{s}}, \\
& C 2: C \geq \bar{C}, \\
& C 3: P_{s} \geq 0 .
\end{array}
$$

Here we have the theorem to show the relationship between the transformed problem and the original problem.

Theorem 1. The optimum energy efficient $q^{*}=\frac{C^{*}}{P_{c}{ }^{*}}=$ $\max _{P_{s}} \frac{C}{P_{c}}$ if and only if $q^{*}$ satisfies

$$
\begin{aligned}
& \max _{P_{s}} C-q^{*} P_{c}, \\
& =C^{*}-q^{*} P_{c}{ }^{*}, \\
& =0 .
\end{aligned}
$$

Proof. Please refer to [19].
By introducing the above theorem, the objective function of the original problem can be be transformed into a subtractive form, which is equivalent to its original one. Since the both problems have the same optimum solution, we solve the transformed problem as follows.

It can be confirmed that $\frac{\partial^{2} C}{\partial^{2} P_{s}}<0$. Therefore, the transformed problem is convex and can be solved by its Largrange dual problem due to the strong duality existing in it. The Lagrangian function of the transformed problem is given by

$$
L\left(\beta, \lambda, P_{s}\right)=C-q^{*} P_{c}-\beta\left(P_{s}-\overline{P_{s}}\right)-\lambda(\bar{C}-C),
$$

where $\beta \geq 0$ and $\lambda \geq 0$ are the Lagrange multipliers corresponding the constraints $C 1$ and $C 2$ in the problem. The dual problem can by expressed by

$$
\min _{\beta, \lambda \geq 0} \max _{P_{s}} L\left(\beta, \lambda, P_{s}\right)
$$

The dual problem can be solved by updating the optimum solution of the transmitted power with Lagrange multipliers. According to KKT conditions with $\frac{\partial L\left(\beta, \lambda, P_{s}\right)}{\partial P_{s}}=0$, the optimum value of $P_{s}$ in the cooperative network with the selfenergy recycling relaying protocol can be obtained as

$$
P_{s}=\left[\frac{1+\lambda}{2 \ln 2\left(q^{*}+\beta\right)}-\frac{\sigma_{r}^{2}}{|h|^{2}}\right]^{+},
$$

or

$$
P_{s}=\left[\frac{1+\lambda}{2 \ln 2\left(q^{*}+\beta\right)}-\frac{\sigma_{d}^{2}-\eta|f|^{2} \sigma_{d}^{2}}{\eta|h|^{2}|g|^{2}}\right]^{+},
$$

There are two close forms of the transmitted power because the different channel state information of the considered cooperative network leads to different formulas to calculate the channel capacity in the DF protocol. Specifically, if $C_{s r}<C_{r d}$, the optimum value of transmitted power is calculated by (16). If not, the optimum value of transmitted power is calculated by (17). Unlike other cooperative networks, the channel capacity of the source-relay link and that of the relay-destination link both are the function of the transmitted power. Therefore we know the formulation to calculate the channel capacity if the channel state information is available.

So far, the optimum $P_{s}$ can be obtained with given Lagrange multipliers and we can use the gradient method in the iteration algorithm to update $\beta$ and $\lambda$, which are given by

$$
\begin{gathered}
\beta(n+1)=\left[\beta(n)-\psi_{\beta}\left(\overline{P_{s}}-P_{s}\right)\right]^{+}, \\
\lambda(n+1)=\left[\lambda(n)-\psi_{\lambda}(C-\bar{C})\right]^{+},
\end{gathered}
$$

where $\psi_{\beta}$ and $\psi_{\lambda}$ are positive step sizes. $n$ is the iteration index. With these updated Lagrange multipliers, the optimum energy efficiency can be obtained. Inspired by the Dinkelbach method [19], the algorithm is proposed as follows

Algorithm: Energy-Efficient optimization Iteration Algorithm

1: Initialize the $q^{*}$, Lagrange multipliers $\beta$ and $\lambda$, the maximum number of iteration $n_{\max }$ and the maximum tolerance $\epsilon$.

2: Obtain $P_{s}$ in one of (16) and (17).

3: Use (18) and (19) to update the $\beta$ and $\lambda$ in the iteration 
procedure.

4: If $C^{*}-q^{*} P_{c}{ }^{*}>\epsilon$, then set $q^{*}=\frac{C^{*}}{P_{c}{ }^{*}}$ and repeat the step 2 and 3. If $C^{*}-q^{*} P_{c}{ }^{*}<\epsilon$, the optimum $P_{s}{ }^{*}$ and $q^{*}$ are obtained.

From the proposed algorithm, we can see that the transformed parametric problem can be solved by the Lagrange multiplier method with the given $q^{*}$. The convergence of the optimum $q^{*}$ in its parametric problem is guaranteed.

Proof. Please refer to Appendix.

\section{NUMERICAL RESULTS}

In this section, simulation results are provided to evaluate the performance of the proposed energy-efficient optimization algorithm. The distances from the source to the relay and the relay to the destination are $10 \mathrm{~m}$ with the path loss exponent $m=3$. The antenna noise variance and conversion noise variance are equal to $-50 \mathrm{dbm}$. The energy harvesting coefficient is set as 0.5. The circuit-consumed power at the source, the relay and the destination is all set as $20 \mathrm{dbm}$. The maximum transmitted power is $30 \mathrm{dbm}$. the minimum required channel capacity is $1 \mathrm{bits} / \mathrm{sec} / \mathrm{Hz}$. The channel between the transmit-receive antenna pair is simulated as $h=(d)^{-\frac{m}{2}} e^{j w}$. The loop channel path loss is $-15 \mathrm{db}$ [20]. Simulation results were averaged over 1000 independent trials.

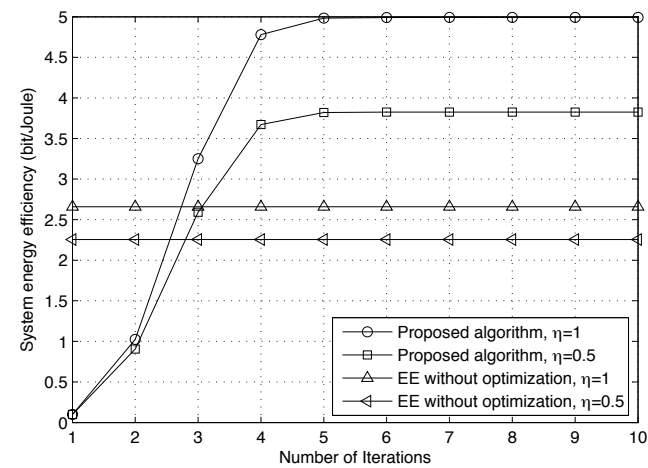

Fig. 1. The performance of proposed algorithm with the different levels of energy conversion coefficient $\eta$ and system performance without energy efficiency optimization

In Fig. 1, the convergence of the proposed algorithm for energy-efficient maximization in cooperative networks with the self-energy recycling relaying protocol is shown, where the energy efficiency for the case without optimization is also provided as a benchmark. As can be seen from the figure, the proposed algorithm converges within 5 iterations. The concavity of problems can be guaranteed. Since the proposed algorithm always converges, the optimum energy efficiency can be obtained by using the proposed algorithm. The Fig. 1 also presents the performance with different levels of the energy conversion coefficient $\eta$. With the bigger $\eta$, the relay node can harvest more power from the source node and its loop-back channel. Therefore the system performance achieves better. To facilitate a better performance evaluation, the energy efficiency without optimization with different $\eta$ is also provided. The value of energy efficiency without the proposed algorithm is obtained with the maximum allowed transmitted power. It is obvious that the energy efficiency can not outperform the proposed algorithm. The reason for this performance gain is following. Recall that the energy efficiency is calculated from the ratio of the channel capacity and the overall power consumption. Increasing the power can achieve larger channel capacity, but it consumes more power. The proposed algorithm guarantees that better energy efficiency is achieved.

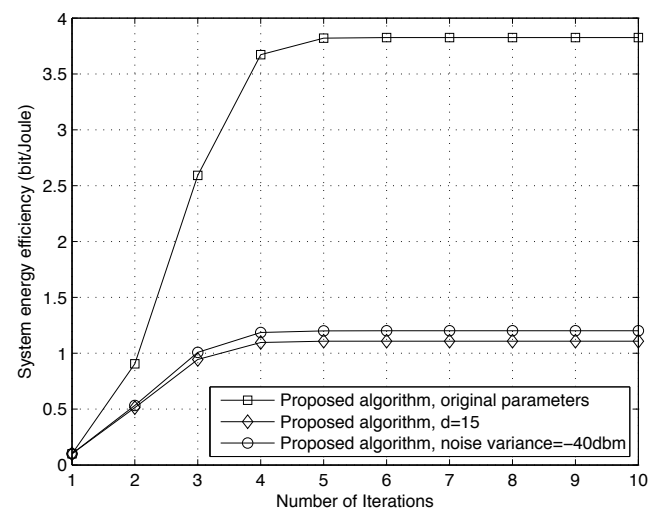

Fig. 2. Energy efficiency optimization for the self-energy recycling relaying protocol with different parameters

Fig. 2 illustrates the performance of system energy efficiency maximization with different noise variance and distance based on the self-energy recycling relaying protocol. As can be seen from the picture, the energy efficiency is deteriorated by the increasing noise variance and distances. The reason is that when noise variance and distance increase, channel capacity will reduce and then lead to the decrease of energy efficiency.

\section{CONCLUSIONS}

This paper proposed feasible solutions for the maximization of energy efficiency in wireless cooperative networks based on a self-energy recycling relaying protocol. The original problem was first transformed to a parametric problem by using nonlinear fractional programming and then solved by applying the Lagrange multiplier method. Simulation results confirm the energy efficiency of the proposed algorithm in the considered model. The trade-off between energy efficiency and system parameters is also analysed by using simulation results.

\section{APPENDIX}

\section{A. Convergence analysis}

We prove that the convergence of the transformed parametric problem can be guaranteed. First of all, we introduce two lemmas.

Lemma 1. The object function $C-q^{*} P_{c}$ is a monotonic decreasing function of $q^{*}$.

Proof. Given two power allocation results $P_{s 1}$ and $P_{s 2}$ with their corresponding energy efficiency $q_{1}^{*}$ and $q_{2}^{*}$. We define 
$q_{1}^{*}>q_{2}^{*}$, then we have

$$
\begin{aligned}
& \max _{P_{s}} C-q_{2}^{*} P_{c}, \\
& =C\left(P_{s 2}\right)-q_{2}^{*} P_{c}\left(P_{s 2}\right), \\
& >C\left(P_{s 1}\right)-q_{2}^{*} P_{c}\left(P_{s 1}\right), \\
& \geq C\left(P_{s 1}\right)-q_{1}^{*} P_{c}\left(P_{s 1}\right), \\
& =\max _{P_{s}} C-q_{1}^{*} P_{c} .
\end{aligned}
$$

Lemma 2. There is an energy efficient solution $q^{*}$ to ensure $C-q^{*} P_{c}=0$.

Proof. It can be proved that the objective function is continuous in $q^{*}$. If $q^{*}$ is plus infinity, the value of the objective function is minus infinity and vice-versa. Hence there is a $q^{*}$ to let $C-q^{*} P_{c}=0$.

With the above lemmas, we can prove the convergence of the transformed parametric problem. We denote $P_{s n}$ and $q_{n}^{*}$ as the energy efficiency policy in the $n$-th iteration. Recall that in the algorithm, $q_{n+1}^{*}=\frac{C\left(P_{s_{n}}\right)}{P_{c}\left(P_{s_{n}}\right)}$. Then we have

$$
\begin{aligned}
& C\left(P_{s n}\right)-q_{n}^{*} P_{c}\left(P_{s n}\right), \\
& =q_{n+1}^{*} P_{c}\left(P_{s n}\right)-q_{n}^{*} P_{c}\left(P_{s n}\right), \\
& =\left(q_{n+1}^{*}-q_{n}^{*}\right) P_{c}\left(P_{s n}\right) .
\end{aligned}
$$

where $P_{c}\left(P_{s n}\right)$ is greater than 0 , therefore $q_{n+1}^{*}-q_{n}^{*} \geq 0$. The $q_{n}^{*}$ is non-decreasing in the iterative process. In lemma 1 , the objective function is monotonic decreasing in $q_{n}^{*}$ and $q_{n}^{*}$ is non-decreasing in the iteration, therefore the objective function is non-increasing in the iteration. In lemma 2 , the optimum energy efficiency converges when $C-q^{*} P_{c}=0$. If the iteration index is big enough, $C-q^{*} P_{c}$ will equal to 0 and the energy efficiency will be obtained.

\section{REFERENCES}

[1] R. Zhang and C. K. Ho, "MIMO broadcasting for simultaneous wireless information and power transfer," IEEE Trans. Wireless Commun. vol. 12, no. 5, pp. 1989-2001, May 2013.

[2] L. Liu, R. Zhang, and K. C. Chua, "Wireless information and power transfer: A dynamic power splitting approach,” IEEE Trans. Commun., to appear in 2013 (available on-line at arXiv:1302.0585).

[3] X. Zhou, R. Zhang, and C. K. Ho, "Wireless information and power transfer: Architecture design and rate-energy tradeoff," IEEE Transactions on Communications, (submitted) Available: http://arxiv.org/abs/1205.0618.

[4] K. Huang and V. K. N. Lau, "Enabling wireless power transfer in cellular networks: architecture, modeling and deployment," IEEE J. Sel. Areas Commum., 2012. Available at http://arxiv.org/abs/1207.5640.

[5] V. Raghunathan, S. Ganeriwal, and M. Srivastava, "Emerging techniques for long lived wireless sensor networks," IEEE Commum. Mag., vol. 44, no. 4, pp. 108-114, Apr. 2006.

[6] J. A. Paradiso and T. Starner, "Energy scavenging for mobile and wireless electronics," IEEE Trans. Pervasive Comput., vol. 4, no. 1, p. 1827, Jan. 2005.

[7] B. Medepally and N. B. Mehta, "Voluntary energy harvesting relays and selection in cooperative wireless networks," IEEE Trans. Wireless Commun., vol. 9, no. 11, pp. 3543-3553, Nov. 2010.

[8] L. R. Varshney, "Transporting information and energy simultaneously," in Proc. IEEE Int. Symp. Inf. Theory (ISIT), Toronto, Canada, Jul. 2008

[9] L. Liu, R. Zhang, and K. C. Chua, "Wireless information transfer with opportunistic energy harvesting," IEEE Trans. Wireless Commun., 2012. Available at http://arxiv.org/abs/1204.2035.
[10] Z. Xiang and M. Tao, "Robust beamforming for wireless information and power transmission," IEEE Wireless Commun. Lett., vol. 1, no. 4, pp. 372-375, 2012.

[11] A. A. Nasir, X. Zhou, S. Durrani, and R. A. Kennedy, "Relaying protocols for wireless energy harvesting and information processing," IEEE Trans. Wireless Commun., to appear in 2013.

[12] Z. Ding, S. M. Perlaza, I. Esnaola, and H. V. Poor, "Power allocation strategies in energy harvesting wireless cooperative networks," IEEE Trans. Wireless Commun., submitted (available at http://arxiv.org/abs/1307.1630).

[13] H. Ju and R. Zhang, "Optimal resource allocation in full-duplex wireless powered communication network," IEEE Trans. Commun., Available at http://arxiv.org/abs/1403.2580.

[14] C. Zhong, H. A. Suraweera, G. Zheng, I. Krikidis, and Z. Zhang, "Wireless information and power transfer with full duplex relaying," IEEE Trans. Commun., Available at http://arxiv.org/abs/1409.3904.

[15] Y. Zneg and R. Zhang, "Full-duplex wireless-powered relay with selfenergy recycling," IEEE Wireless Commun. Letters, vol. PP, p. 1, Jan. 2015.

[16] D. W. K. Ng, E. Lo, and R. Schober, "Energy-efficient resource allocation in OFDMA systems with large numbers of base station antennas," IEEE Trans. Wireless Commun., vol. 11, pp. 3292-3304, Sep. 2012.

[17] T. Chen, Y. Yang, H. Zhang, H. Kim, and K. Horneman, "Network energy saving technologies for green wireless access networks," IEEE Wireless Commun., vol. 18, pp. 30-38, Oct. 2011.

[18] C. Zarakovitis and Q. Ni, "Maximising energy efficiency in multi-user multi-carrier broadband wireless systems: convex relaxation and global optimisation techniques," IEEE Trans. on Vehicular Technology, 2015, DOI: $10.1109 /$ TVT.2015.2455536.

[19] W. Dinkelbach, "On nonlinear fractional programming," Management Science, vol. 13, pp. 492-498, Mar. 1967.

[20] H. G. Schantz, "Near field propagation law and a novel fundamental limit to antenna gain versus size," IEEE Antennas and Propag. Society Int. Symposium, Jul. 2005. 